\title{
ESTUDO QUÍMICO DE UMA AMOSTRA DE PRÓPOLIS VERDE DE PASSA QUATRO, MINAS GERAIS, BRASIL\#
}

Leonardo Carvalho Tavares, Telma Leda Gomes de Lemos e Angela Martha Campos Arriaga*

Departamento de Química Orgânica e Inorgânica, Centro de Ciências, Universidade Federal do Ceará, CP 12200, 60021-970

Fortaleza - CE, Brasil

Gilvandete Maria Pinheiro Santiago

Departamento de Farmácia, Universidade Federal do Ceará, Rua Capitão Francisco Pedro 1210, 60430-370 Fortaleza - CE, Brasil Raimundo Braz-Filho

Fundação de Amparo à Pesquisa do Estado do Rio de Janeiro / LCQUI, CCT, Universidade Estadual do Norte Fluminense, 28013603 Campos dos Goytacazes - RJ, Brasil

Recebido em 18/5/10; aceito em 3/9/10; publicado na web em 25/10/10

CHEMICAL STUDY OF A SAMPLE OF GREEN PROPOLIS FROM PASSA QUATRO, MINAS GERAIS-BRAZIL. The phytochemical investigation of a sample of propolis from Passa Quatro-Minas Gerais State, Brazil, where nine constituents were isolated: a mixture of $\alpha$ - and $\beta$-amyrin, lupeol, a mixture of flavonols ramnocitrin and eupalitin, acacetin, 3-prenyl-4-hydroxycinnamic acid, 3,5-diprenyl-4-hydroxycinnamic acid the new compound, the (E)-3-[4-(3-phenylpropanoiloxy)]-3,5-diprenil-cinnamic acid. The structures of the isolated compounds were characterized by 1D- and 2D-NMR experiments, MS and IR spectrometry, and comparison with data described in the literature.

Keywords: propolis; (E)-3-[4-(3-phenylpropanoiloxy)]-3,5-diprenyl-cinnamic acid; cinnamic acid derivatives.

\section{INTRODUÇÃO}

O termo própolis significa defesa da cidade ou da colmeia (pro "em defesa de" e polis "cidade"). ${ }^{1,2}$ As abelhas, de fato, usam esta substância para protegê-las contra insetos e micro-organismos empregando-a em finas camadas nas paredes internas das colmeias, para vedar aberturas e rachaduras, reparar e fortalecer os favos de mel, no preparo de locais assépticos para a postura da abelha rainha e na mumificação de insetos invasores. ${ }^{3}$

A própolis é conhecida e utilizada pelo homem desde os tempos mais remotos; os sacerdotes do antigo Egito a utilizavam frequentemente como substância medicinal e como parte integrante dos unguentos e cremes para embalsamar; persas, romanos e incas também fizeram uso da própolis para tratamento de infecções. ${ }^{4}$ Dependendo da flora de origem e da idade, sua coloração varia do marrom escuro, passando a uma tonalidade esverdeada até o marrom avermelhado e seu odor é variável de uma amostra para outra. ${ }^{1,5}$

Particularmente, sob a forma de extratos hidroetanólicos, a própolis vem se destacando tanto pelas suas propriedades terapêuticas, tais como atividades antimicrobiana, anti-inflamatória, cicatrizante, anestésica, anticancerígena e fotoprotetora, ${ }^{6}$ quanto pela possibilidade de aplicação em indústrias farmacêutica e alimentícia, na forma de alimentos funcionais. ${ }^{7,8}$

A própolis verde, produzida em Minas Gerais principalmente a partir de Baccharis dracunculifolia DC (Asteraceae), tem sido relatada na literatura como potencializadora da resposta imunológica, ${ }^{9}$ como anti-inflamatória, ${ }^{10,11}$ inibidora do crescimento tumoral ${ }^{11} \mathrm{e}$ hepatoprotetora. ${ }^{12}$ Seu constituinte majoritário é o ácido 3,5-diprenil4-hidroxicinâmico (artepilina), ${ }^{11}$ sendo também fonte de outros derivados prenilados do ácido $p$-cumárico, e de grande quantidade de flavonoides, muitos dos quais não estão presentes na própolis da Europa, América do Norte e Ásia. ${ }^{7}$ Amostras de própolis de outras regiões do Brasil também têm sido estudadas quanto a sua constituição

*e-mail: angelamcarriaga@yahoo.com.br

\# Artigo em homenagem ao Prof. Hans Viertler química e à influência da sazonalidade em atividades biológicas e na composição fenólica. ${ }^{13}$

Este trabalho focalizou uma reinvestigação fitoquímica de uma amostra de própolis verde, obtida na cidade de Passa Quatro (Minas Gerais). Do extrato etanólico foram isolados e caracterizados a mistura de $\alpha$ - e $\beta$-amirinas (1a e 1b), o lupeol (2), a mistura dos flavonóis ramnocitrina (3a) e eupalitina (3b), acacetina (4) e os ácidos 3-prenil-4-hidroxicinâmico (5), artepilina (6) e a nova substância caracterizada como o ácido (E)-3-[4-(3-fenilpropanoiloxi)]-3,5diprenil-cinâmico (7).

\section{RESULTADOS E DISCUSSÃO}

A mistura dos triterpenos 1a e 1b, bem como a substância $\mathbf{2}$, foram identificadas através da análise dos espectros no IV, RMN de ${ }^{1} \mathrm{He}{ }^{13} \mathrm{C}$ e comparação com dados da literatura. ${ }^{14}$

O espectro no IV da substância $\mathbf{3}$ apresentou bandas compatíveis com a presença de hidroxila fenólica e carbonila conjugada. Os dados dos espectros de RMN de ${ }^{13} \mathrm{C}\left(\left\{{ }^{1} \mathrm{H}\right\}\right.$ e DEPT) de $\mathbf{3}$ foram usados para caracterização de sistema flavonoídico e para reconhecer a presença de uma mistura binária (3a e 3b) através da duplicidade de alguns sinais. Observaram-se também sinais indicativos de carbonos carbonílicos $\left(\delta_{\mathrm{C}} 177,87\right.$ e 177,61) com ligação de hidrogênio e em $\delta_{\mathrm{C}} 61,14$ indicativo de metoxila localizada em posição estericamente impedida. $\mathrm{O}$ espectro de $\mathrm{RMN}$ de ${ }^{1} \mathrm{H}$ revelou sinais de hidrogênios aromáticos em $\delta_{\mathrm{H}} 8,16(\mathrm{~d}, J=8,5 \mathrm{~Hz})$ e $7,04(\mathrm{~d}, J=8,5 \mathrm{~Hz})$, sugerindo a presença de um sistema do tipo AA 'BB' de anel aromático para-dissubstituído, e simpletos em $\delta 6,50, \delta 6,40$ e $\delta 6,19$. Sinais simples em $\delta 3,87$ $(3 \mathrm{H})$ e 3,85 (6H) foram atribuídos a hidrogênios de três grupamentos metoxílicos. Estas deduções, a comparação com dados descritos na literatura ${ }^{12,13} \mathrm{e}$ a análise dos espectros 2D HMQC e HMBC permitiram identificar os componentes da mistura $\mathbf{3}$ como ramnocitrina (3a) e eupalitina (3b). Assim, $\mathbf{3}$ foi caracterizada como uma mistura de $\mathbf{3 a}$ e 3b, flavonóis já isolados de Trichogonia menthaefolia e Mikania micrantha ${ }^{15,16}$ As percentagens aproximadas dos dois componentes da mistura foram deduzidas como sendo $34,5 \%$ de $\mathbf{3 a}$ e $65,5 \%$ de 
$\mathbf{3 b}$, através das integrações dos sinais correspondentes aos átomos de hidrogênio aromático $\mathrm{H}-8$ presentes no espectro de $\mathrm{RMN}$ de ${ }^{1} \mathrm{H}$.

Os dados espectrais das substâncias 4, 5 e 6, principalmente RMN de ${ }^{1} \mathrm{H}$ e ${ }^{13} \mathrm{C}\left(\left\{{ }^{1} \mathrm{H}\right\}\right.$ e DEPT $)$ 1D e $2 \mathrm{D}\left({ }^{1} \mathrm{H}-{ }^{1} \mathrm{H}-\mathrm{COSY}, \mathrm{HMQC}\right.$ e HMBC $)$ e massas, e comparação com valores descritos na literatura foram utilizados para identificar $\mathbf{4}$ como a flavona acacetina, ${ }^{17} \mathbf{5}$ como ácido 3-prenil-4-hidroxicinâmico ${ }^{4,18,19}$ e 6 como ácido (E)-3,5-diprenil-4hidroxicinâmico, conhecido também pelo o nome comum artepilina. ${ }^{11,18}$

O composto 7 apresentou-se como líquido viscoso. O espectro no IV evidenciou bandas compatíveis com hidroxila $\left(v_{\max } 3442 \mathrm{~cm}^{-1}\right)$, com esqueleto aromático $\left(v_{\max } 1594 \mathrm{~cm}^{-1}\right)$ e com a presença de carbonilas de ácido $\alpha, \beta$-insaturado $\left(\nu_{\max } 1693 \mathrm{~cm}^{-1}\right)$ e de éster $\left(\nu_{\max } 1700 \mathrm{~cm}^{-1}\right)$. $\mathrm{O}$ espectro de massas de alta resolução de 7 , registrado no modo negativo, revelou pico correspondente ao íon quasi molecular em $m / z$ 431,2202 ([M-H $]^{+}$, calculado: $\left.m / z, 431,2222\right)$ e permitiu deduzir a sua fórmula molecular como $\mathrm{C}_{28} \mathrm{H}_{32} \mathrm{O}_{4}$. Seu espectro de $\mathrm{RMN}$ de ${ }^{13} \mathrm{C}$ apresentou quatorze sinais que foram atribuídos a vinte átomos de carbono insaturados entre $\delta_{\mathrm{C}} 114,24$ a 179,17 (Tabela 1), sete a mais que a substância $\mathbf{6}$.

Os espectros de RMN de ${ }^{13} \mathrm{C}\left(\left\{{ }^{1} \mathrm{H}\right\}\right.$ e DEPT) apresentaram, além de sinais relativos aos carbonos do anel aromático, dois sinais na região de carbonos carbonílicos $\left(\delta_{C} 173,05\right.$ e 179,17), três sinais atribuídos a quatro carbonos metilênicos $\mathrm{sp}^{3}: \delta_{C} 30,81$ (C-7"), 35,82 (C-8") e 29,66 (C-1'/ C-1"')] e dois representando quatro carbonos metílicos: $\delta_{C} 18,09$ (C-4'/ C-4"') e $\delta_{C} 26,05$ (C-5'/ C-5")], característicos de duas unidades prenílicas. Os dados de $\mathrm{RMN}$ de ${ }^{13} \mathrm{C}$ sugeriram também a existência de dois anéis aromáticos devido aos sinais em $\delta_{\mathrm{C}} 140,41$ (C-1"'”), 128,28 (C-2"'/C-6"'), 128,79 (C-3"'/C-5"'), 127,31 (C-4"”), 131,29 (C-1), 126,56 (C-2/C-6), 130,18 (C-3/C-5) e 155,65 (C-4). Em adição, a presença de carbonos de uma ligação dupla dissubstituída em $\delta$ 147,71 (CH-7) e $\delta$ 114,24 (CH-8) e uma carbonila de ácido carboxílico em $\delta 173,05$ (C-9) apontou para uma estrutura que teria o ácido cinâmico como núcleo central, de maneira semelhante às substâncias $\mathbf{5}$ e $\mathbf{6}$. Sinais característicos de unidade prenila, como já descritas anteriormente, foram também observadas, indicando semelhança estrutural entre as três substâncias; entretanto, observou-se em 7 a presença de um carbono aromático oxigenado em $\delta_{\mathrm{C}} 155,65$, provavelmente esterificado, hipótese fortemente apoiada pelo sinal em $\delta 179,17$ (C-9') compatível com carbonila de éster, apontando que 7 teria alguma semelhança estrutural com a baccharina [ácido 3-prenil-4-(2,3-di-hidrocinamoil) cinâmico], já isolada da própolis verde. ${ }^{20} \mathrm{~A}$ análise do espectro de $\mathrm{RMN}$ de ${ }^{1} \mathrm{H}$ de 7 corroborou com as deduções discutidas, indicando, além dos sinais referentes às unidades diprenílicas da molécula, a presença de hidrogênios aromáticos através dos sinais entre $\delta_{\mathrm{H}} 7,20-7,25(3 \mathrm{H}, \mathrm{m})$ e $\delta 7,32(2 \mathrm{H}, \mathrm{t}, J=7,3 \mathrm{~Hz})$. Estes sinais e a presença de dois tripletos referentes a hidrogênios de carbonos metilênicos em $\delta 2,99$ (t, $J=$ $7,2 \mathrm{~Hz}, 2 \mathrm{H})$ e $\delta 2,72(\mathrm{t}, J=7,2 \mathrm{~Hz}, 2 \mathrm{H})$ permitiram caracterizar uma unidade 3- fenilpropanoiloxi (Figura 1).

A comparação dos dados de $\mathrm{RMN}$ de ${ }^{1} \mathrm{H}$ e ${ }^{13} \mathrm{C}$ de 7 e 6 e os espectros 2D HSQC $\left({ }^{1} J_{\mathrm{CH}}\right)$ e HMBC $\left({ }^{\mathrm{n}} J_{\mathrm{CH}}, \mathrm{n}=2\right.$ e 3$)$ de 7 (Tabela 1) revelaram estar em plena concordância com todas estas deduções, sendo o HMBC usado também para assegurar a localização dos substituintes nos anéis aromáticos. As correlações a três ligações $\left({ }^{3} J\right)$ entre os hidrogênios $2 \mathrm{H}-1$ '/2H-1" $\left(\delta_{\mathrm{H}} 3,37\right)$ com os carbonos CH-2/CH-6 $\left(\delta_{\mathrm{C}} 126,56\right)$ e C-4 $\left(\delta_{\mathrm{C}} 155,65\right)$ e H-7 $\left(\delta_{\mathrm{H}} 7,71\right)$ do sistema $\alpha, \beta$-insaturado com $\mathrm{CH}-2 / \mathrm{CH}-6\left(\delta_{\mathrm{C}} 126,56\right)$ asseguraram o padrão de substituição do anel aromático presente no sistema $(E)$-cinâmico $(J$ $=15,8 \mathrm{~Hz}$ ) contendo átomo de oxigênio no carbono em C-4 (Tabela 1). Outras interações heteronucleares à longa distância $\left({ }^{\mathrm{n}} J_{\mathrm{CH}}, \mathrm{n}=2 \mathrm{e}\right.$ 3) observadas no HMBC encontram-se registradas na Tabela 1, inclusive aquelas envolvendo a unidade 3-fenilpropanoiloxi da função éster. Estas informações permitiram caracterizar o composto 7 como<smiles>[R]Oc1c([R])cc(/C=C/C(=O)O)cc1CC=C(C)C</smiles>

$5 \mathrm{R}=\mathrm{R}_{1}=\mathrm{H}$<smiles>[R]C=C([18F])[13CH]C[R]</smiles>

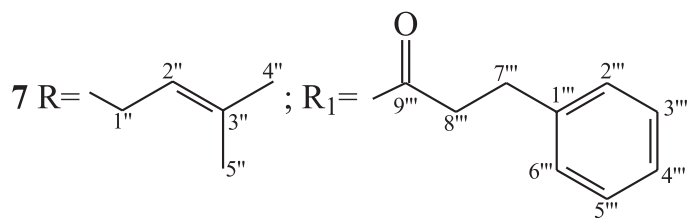

Figura 1. Constituintes 5, 6 e 7 isolados de Própolis Verde de Passa Quatro, Minas Gerais- Brasil

ácido (E)-3-[4-(3-fenilpropanoiloxi)]-3,5-diprenil-cinâmico que, no melhor de nosso conhecimento, está sendo descrito pela primeira vez.

\section{PARTE EXPERIMENTAL}

\section{Procedimentos experimentais gerais}

Os espectros de absorção na região do infravermelho (IV) foram obtidos em espectrômetro Perkin Elmer, modelo FT-IR Spectrum 1000, usando-se pastilhas de $\mathrm{KBr}$. Os espectros RMN de ${ }^{1} \mathrm{H} \mathrm{e}{ }^{13} \mathrm{C}$ foram registrados em espectrômetro de Bruker, modelo DRX-500, operando na frequência de $125 \mathrm{MHz}$ para ${ }^{13} \mathrm{C}$ e $500 \mathrm{MHz}$ para ${ }^{1} \mathrm{H}$, usando-se $\mathrm{CDCl}_{3}$ e $\mathrm{CD}_{3} \mathrm{OD}$ como solventes. Como padrão interno para referência de deslocamento químico, $\delta$, foi usado o tetrametilsilano. Os espectros de massa de baixa e alta resolução foram obtidos, respectivamente, em espectrômetros Shimadzu QP505A operando em 70 eV e Shimadzu LCMS-TOF (225-07100-34) equipado com fonte de ionização de electronspray.

Nas análises cromatográficas de adsorção e exclusão molecular em colunas foram utilizados gel de sílica (230-400 e 70-230 mesh) da Vetec ou Aldrich, e Sephadex LH-20 como fases estacionárias, respectivamente. O comprimento e o diâmetro das colunas foram estabelecidos com base nas quantidades das amostras. Na cromatografia em camada delgada (CCD) foram usadas cromatoplacas de gel de sílica $60 \mathrm{PF}_{254}$ (Sorbent) e como reveladores foram usados vapores de iodo e/ou vanilina.

\section{Coleta de material}

A amostra de própolis verde utilizada foi produzida pela espécie Apis mellifera, cedida pela pesquisadora Dra. C. A. Dorneles, coletada na cidade de Passa Quatro, Minas Gerais, Brasil em outubro de 2005, e mantida sob refrigeração a aproximadamente $4{ }^{\circ} \mathrm{C}$.

\section{Extração e isolamento dos constituintes}

Uma amostra de $5 \mathrm{~kg}$ de própolis verde, fragmentada em pequenas porções, foi extraída exaustivamente com etanol e, após evaporação do solvente a vácuo, forneceu o extrato etanólico (1,2 kg). Esse extrato foi solubilizado em etanol $(300 \mathrm{~mL})$ e particionado com éter de 
Tabela 1. Dados de RMN de ${ }^{1} \mathrm{H}(500 \mathrm{MHz})$ e de $\mathrm{RMN}$ de ${ }^{13} \mathrm{C}(125 \mathrm{MHz})$ do composto 7 em comparação com dados de RMN do composto 6

\begin{tabular}{|c|c|c|c|c|c|c|}
\hline & & & 7 & & & \\
\hline & & QC & & IBC & & QC \\
\hline & $\delta_{\mathrm{C}}$ & $\delta_{\mathrm{H}}$ & ${ }^{2} J_{\mathrm{CH}}$ & ${ }^{3} J_{\mathrm{CH}}$ & $\delta_{\mathrm{C}}$ & $\delta_{\mathrm{H}}$ \\
\hline $\mathbf{C}$ & & & & & & \\
\hline 1 & 131,29 & - & H-2/H-6 & $\mathrm{H}-8$ & 126,60 & - \\
\hline 3,5 & 130,18 & - & $\begin{array}{c}\text { H-2/H-6; } \\
\text { H-1'”/H-1,', }\end{array}$ & & 127,90 & - \\
\hline 4 & 155,65 & - & & H-2/H-6; H-1'/H-1', & 155,64 & - \\
\hline 9 & 173,05 & - & $\mathrm{H}-8$ & $\mathrm{H}-7$ & 171,87 & - \\
\hline $1 "$, & 140,41 & - & $\mathrm{H}-7^{\prime \prime}$, & H-3"'/H-5" '; H-8," & - & - \\
\hline 3,3, & 135,33 & - & $\begin{array}{l}\text { H-4'/H-4'”; } \\
\text { H-5'/H-5', }\end{array}$ & H-1'/H-1', & 135,37 & - \\
\hline $9, "$ & 179,17 & - & $\mathrm{H}-8^{\prime \prime}$ & $\mathrm{H}-7, "$ & - & - \\
\hline $\mathbf{C H}$ & & & & & & \\
\hline 2,6 & 126,56 & $7,25-7,20(\mathrm{~m})$ & & H-7; H-1'/H-1'” & 128,60 & $7,22(s)$ \\
\hline 7 & 147,71 & $7,72(\mathrm{~d}, 15,8)$ & & H-2/H-6 & 147,59 & $7,70(\mathrm{~d}, 15,8)$ \\
\hline 8 & 114,24 & $6,30(\mathrm{~d}, 15,8)$ & & & 114,12 & $6,30(\mathrm{~d}, 15,8)$ \\
\hline $2^{\prime}, 2^{\prime \prime}$ & 121,50 & $5,33(\mathrm{t}, 6,6)$ & H-1'/H-1', & H-4'/H-4”; H-5'/H-5" & 121,52 & $5,33(\mathrm{tl})$ \\
\hline $2 ",, 6 "$ & 128,28 & $7,25-7,20(\mathrm{~m})$ & H-3""'/H-5", & $\mathrm{H}-7, "$ & - & - \\
\hline $3 ", 5 "$, & 128,79 & $7,32(\mathrm{t}, 7,3)$ & & & - & - \\
\hline $4 "$, & 127,31 & $7,25-7,20(\mathrm{~m})$ & & & - & - \\
\hline $\mathrm{CH}_{2}$ & & & & & & \\
\hline $1^{\prime}, 1^{\prime \prime}$ & 29,66 & $3,37(\mathrm{t}, 7,2)$ & & H-2/H-6 & 29,70 & $3,37(\mathrm{t}, 6,3)$ \\
\hline $7 "$, & 30,81 & $2,99(\mathrm{t}, 7,2)$ & H-8," & H-2"”/H-6", & - & - \\
\hline $8 "$, & 35,82 & $2,72(\mathrm{t}, 7,2)$ & $\mathrm{H}-7^{\prime \prime}$ & & - & - \\
\hline $\mathrm{CH}_{3}$ & & & & & & \\
\hline $4^{\prime}, 4^{\prime \prime}$ & 18,09 & $1,79(\mathrm{sl})$ & & $\begin{array}{l}\text { Н-2'/H-2''; } \\
\text { Н-5'/H-5', }\end{array}$ & 18,17 & $1,78(\mathrm{sl})$ \\
\hline $5,, 5^{\prime \prime}$ & 26,05 & $1,81(\mathrm{sl})$ & & $\begin{array}{l}\text { H-2'/H-2''; } \\
\text { H-4'/H-4', }\end{array}$ & 25,03 & $1,79(\mathrm{sl})$ \\
\hline
\end{tabular}

*Deslocamentos químicos em $\delta$ (ppm), constantes de acoplamento ( $J$ em Hz) de 7 e comparação com os de $\mathbf{6}$.

petróleo, seguido de éter etílico, fornecendo, respectivamente, extratos denominados PEP (330 g) e PEE (457 g). PEE (120 g) foi submetido a um fracionamento em gel de sílica sob pressão reduzida utilizando $200 \mathrm{~mL}$ de cada eluente (hexano, clorofórmio, acetato de etila e metanol), fornecendo os extratos PEH (3 g), PEC (65 g), PEA (31 g) e PEM ( 8 g), respectivamente. A fração PEH foi submetida a sucessivas colunas cromatográficas em gel de sílica, tendo como eluentes misturas binárias de hexano e acetato de etila em ordem crescente de polaridade, que levaram ao isolamento de $\alpha$ - e $\beta$-amirinas (1a e $\mathbf{1 b}$, $26 \mathrm{mg}$ ), de lupeol (2,19 mg), de ácido 3-prenil-4-hidroxicinâmico (5, $23 \mathrm{mg})$, de ácido 3,5-diprenil-4-hidroxicinâmico (6, $8 \mathrm{mg})$ e de ácido (E)-3-[4-(3-fenilpropanoiloxi)]-3,5-diprenil-cinâmico (7, $30 \mathrm{mg}$ ).

\section{Extração e isolamento dos flavonoides}

Uma alíquota da fração PEC (3,0 g) foi dissolvida em uma solução de $\mathrm{NaHCO}_{3} 5 \%$ e posteriormente extraída com acetato de etila. A fração aquosa contendo os sais dos compostos fenólicos foi acidificada com $\mathrm{HCl}$ concentrado até $\mathrm{pH}$ 4. Em seguida, a solução foi extraída com acetato de etila e a fase orgânica concentrada sob pressão reduzida, fornecendo a fração PCC-F $(2,1 \mathrm{~g})$ contendo os compostos fenólicos. Essa fração foi submetida a sucessivas colunas de exclusão com Sephadex LH-20 usando-se metanol como eluente, e forneceu uma mistura de flavonóis [ramnocitrina (3a) e eupalitina (3b), $16 \mathrm{mg}$ ] e a acacetina $(\mathbf{4}, 23 \mathrm{mg})$.

\section{MATERIAL SUPLEMENTAR}

Os espectros no IV, de RMN e massas estão disponíveis em http:// quimicanova.sbq.org.br, em arquivo PDF, com acesso livre.

\section{AGRADECIMENTOS}

Ao CNPq, à CAPES, FUNCAP e FAPERJ pelas bolsas e auxílios financeiros concedidos, ao Centro Nordestino de Aplicação e Uso de Ressonância Magnética Nuclear, Programa de Pós-graduação em Química, Depto. de Química Orgânica e Inorgânica, Centro de Ciências, Universidade Federal do Ceará, Fortaleza - Ceará pelos espectros 1D e 2D de Ressonância Magnética Nuclear (RMN de ${ }^{1} \mathrm{H}$ e RMN de $\left.{ }^{13} \mathrm{C}\right)$.

\section{REFERÊNCIAS}

1. Burdock, G. A.; Food Chem. Toxicol. 1998, 36, 347.

2. Marcucci, M. C.; Quim. Nova 1996, 19, 529. 
3. Bankova, V. S.; Castro, S. L. D.; Marcucci, M. C.; Apidologie 2000, 31, 3.

4. Pereira, A. D. S.; Seixas, F. R. M.; de Aquino Neto, F. R.; Quim. Nova 2002, 25, 321 .

5. Banskota, A. H.; Tezuka, Y.; Adnyana, I. K.; Midorikawa, K.; Matsushige, K.; Message, D.; Huertas, A. A. G.; Kadota, S.; J. Ethnopharmacol. 2000, 72, 239.

6. Reis, G. S.; Valadão, A. F.; Lima, L. R. P.; Moreira, M. L.; Bol. Latinoam. Caribe Plant. Med. Aromat. 2009, 8, 282.

7. Simões, L. C. M.; Gregorio, L. E.; Da Silva Filho, A. A.; De Souza, M. L.; J. Ethnopharmacol. 2004, 94, 59.

8. Ackerman, T.; Food Chem. 1991, 42, 135; Park, Y. K.; Koo, M. H.; Abreu, J. A.; Ikegaki, M.; Cury, J. A.; Rosalen, P. L.; Curr. Microbiol. 1998, 36, 24; Castro, L. M.; Do Nascimento, A. M.; Ikegaki, M.; CostaNeto, C. M.; Alencar, S. M.; Rosalen, P. L.; Bioorgan. Med. Chem. 2009, 17, 5332; Simões-Ambrosio, L. M. C.; Gregório, L. E.; Sousa, J. P. B.; Figueiredo-Rinhel, A. S. G.; Azzolini, A. E. C. S.; Bastos, J. K.; Lucisano-Valim, Y. M.; Fitoterapia 2010, no prelo.

9. Fischer, G.; Cleff, M. B.; Dummer, L. A.; Paulino, N.; Paulino, A. S.; Vilela, C. O.; Campos, F. S.; Storch, T.; Vargas, G. D.; Hübner, S. O.; Vidor, T.; Vet. Immunol. Immunopathol. 2007, 116, 79.

10. Tani, H.; Hasumi, K.; Tatefuji, T.; Hashimoto, K.; Koshino, H.; Takahashi, S.; Bioorg.Med. Chem. 2010, 18, 151.

11. Lemos, M.; Barros, M. P.; Sousa, J. P. B.; Da Silva-Filho, A. A..; Bastos, J. K.; Andrade, S. F.; J. Pharm. Pharmacol. 2007, 59, 603.
12. Banskota, A. H.; Tezuka, Y.; Adnyana, I. K.; Ishii, E.; Midorikawa, K.; Matsushige, K.; Kadota, S.; Phytomedicine 2001, 8, 16.

13. Torres, R. N. S.; Lopes, J. A. D.; Neto, J. M. M.; Citó, A. M. G. L.; Quim. Nova 2008, 31, 479; Cabral, I. S. R.; Oldoni, T. L. C.; Prado, A.; Bezerra, R. M. N.; Alencar, S. M.; Ikegaki, M.; Rosalen, P. L.; Quim. Nova 2009, 32, 1523; Castro, M. L.; Cury, J. A.; Rosalen, P. L.; Alencar, S. M.; Ikegaki, M.; Duarte, S.; Koo, H.; Quim. Nova 2007, 30, 1512.

14. Mahato, S. B.; Kundu, A. P.; Phytochemistry 1994, 37, 1517.

15. Borges, E. M.; Dissertação de Mestrado, Universidade de São Paulo, Brasil, 2006.

16. Wei, X.; Huang, H.; Wu, P.; Cao, H.; Ye, W.; Biochem. Syst. Ecol. 2004, 32, 1091

17. Albuquerque, I. L.; Alves, L. A.; Lemos, T. L. G.; Monte, F. J. Q.; BrazFilho, R.; Quim. Nova 2007, 30, 828.

18. Paulino, N.; Abreu, S. R. L.; Uto, Y.; Koyama, D.; Nagasawa, H.; Hori, H.; Dirsch.V. M.; Vollmar, A. M.; Scremin A.; Bretz, W. A.; Eur. J. Pharmacol. 2008, 587, 296.

19. Marcucci, M. C.; Ferreres, F.; García-Viguera, C.; Bankova, V. S.; De Castro, S. L.; Dantas, A. P.; Vavente, P. H. M.; Paulino, N.; J. Ethnopharmacol. 2001, 74, 105.

20. Sousa, J. P. B.; Silva Filho, A. A.; Bueno, P. C. P.; Gregório, L. E.; Furtado, N. A. J. C.; Jorge, R. F.; Bastos, J. K.; Phytochem. Anal. 2009, 20,24 . 